\title{
ASSOCIATION OF PROTEIN-LOSING ENTEROPATHY CAUSED BY EOSINOPHILIC GASTROENTERITIS WITH ESSENTIAL THROMBOCYTOSIS: CASE REPORT
}

Márcio Silva Miguel Lima, Valdinélia dos Santos Bomfim, Audrey Zeinad, Bruno Ctenas, and Milton Hideaki Arai

Protein-losing enteropathy is not a single disease; it is an atypical manifestation of other disease processes and is marked by excessive loss of protein into the gastrointestinal tract. ${ }^{1}$ These diseases can be classified into 3 large groups according to the protein-loss mechanism: ulcerated lesions with protein exudation (eg, peptic ulcer, inflammatory bowel disease, and gastric carcinoma), mucosal lesions without ulceration, but with increased permeability (eg, Ménétrier's disease, celiac sprue, and eosinophilic gastroenteritis), and obstructive diseases (eg, lymphoma), as well as primary disorder of the lymphatic system (eg, intestinal lymphangiectasia).

One of the several causes of protein-losing enteropathy is eosinophilic gastroenteritis. This is a rare disease characterized by infiltration of eosinophils from the mucosa to the serosa layer, mainly in the stomach and the small bowel. ${ }^{2-4}$ Eosinophilia and anemia are common hematological manifestations, but platelet abnormalities have not been reported.

Essential thrombocytosis is a rare myeloproliferative disorder. High platelet count, which can be as high as more than 1 million platelets $/ \mathrm{mm}^{3}$ in peripheral blood, is the hallmark of this disorder. The disease can progress with thrombotic and hemorrhagic episodes. ${ }^{5}$ Essential thrombocytosis is not a cause of protein-losing enteropathy, and its association with eosinophilic gastroenteritis has not been reported.

We report a rare case of a concurrent manifestation of eosinophilic gastroenteritis and essential thrombocythemia.

A 56-year-old brown-skinned man from São Paulo was admitted to Hospital das Clínicas, the São Paulo University Medical School Hospital in January 2004, with anasarca and ischemia of the fourth and fifth toes of his left foot. The patient reported progressive increase of abdominal girth and lower extremities as well as facial edema for

Hospital das Clínicas, São Paulo University Medical School. Email: marciosml@hotmail.com
5 months, and pain in and darker skin of the fourth and fifth toes of his left foot over the previous 10 days. He also reported hyporexia and a $18-\mathrm{kg}$ body weight loss associated with dyspepsia, flatulence, and episodes of hiccups. He had no complaints of urinary alterations, dyspnea, abdominal pain, intermittent claudication, altered bowel habits, or altered stool.

The patient also reported that he had been hypertensive for 10 years and had made irregular use of propranolol. A former smoker (from 14 to 32 years of age), he had allergic rhinitis.

He had no history of diabetes, excessive alcohol consumption, or blood transfusions.

On physical examination, his overall condition was good, with normal colored mucosa; eupnea; body weight, $70 \mathrm{~kg}$; heart rate, 80 beats/minute; and blood pressure, 150/ $100 \mathrm{~mm} \mathrm{Hg}$. There was evidence of pleural effusion at the base of the left lung, moderate ascites, moderate edema of the lower extremities, and mild facial edema. The fourth and fifth toes of his left foot were sore, with darker coloration (Fig. 1). His peripheral pulses were symmetric with normal amplitudes.

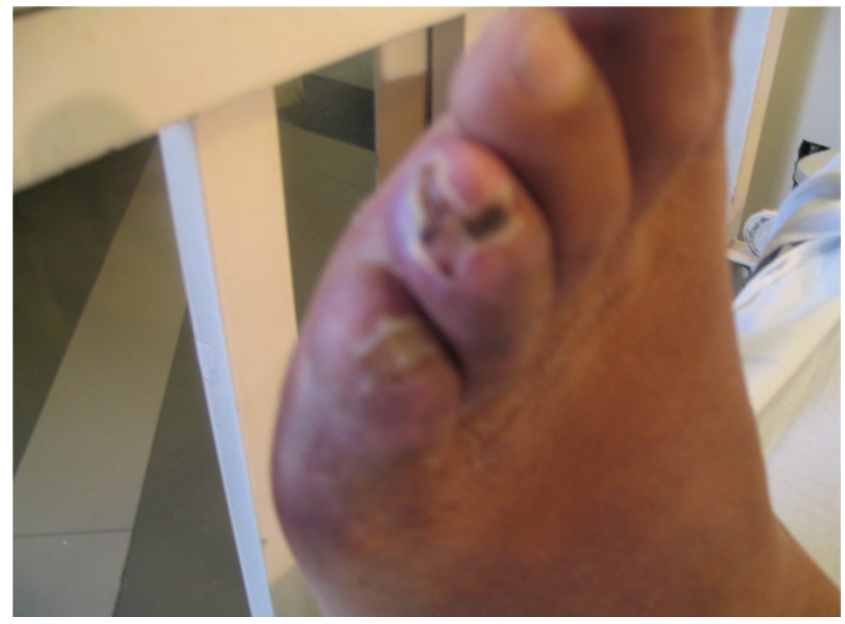

Figure 1 - Gangrene of the left foot toes 
Aspirin, $300 \mathrm{mg} / \mathrm{day}$, pentoxifylline 1,200 mg/day, and analgesics were given, and the clinical condition was investigated. Laboratory tests showed severe hypoalbuminemia ( $\mathrm{alb}=1.8 \mathrm{~g} / \mathrm{dL}$ ), dyslipidemia (total cholesterol $=426 \mathrm{mg} / \mathrm{dL}, \mathrm{LDL}=347 \mathrm{mg} / \mathrm{dL}, \mathrm{HDL}=27 \mathrm{mg} /$ $\mathrm{dL}$ and triglycerides $=259 \mathrm{mg} / \mathrm{dL})$, thrombocytosis $(1.2$ million platelets $/ \mathrm{mm}^{3}$ ), and inflammatory activity (ESR = $85 \mathrm{~mm} / \mathrm{h}, \mathrm{CRP}=66 \mathrm{mg} / \mathrm{dL}$ ). Other results were: $\mathrm{BUN}=$ $39 \mathrm{mg} / \mathrm{dL}$, creatinine $=1.5 \mathrm{mg} / \mathrm{dL}$, hemoglobin $=12.2 \mathrm{mg} /$ $\mathrm{dL}$, hematocrit $=39 \%$, white-cell count $=8,750 / \mathrm{mm}^{3}$ (lymphocytes $=1,800 / \mathrm{mm}^{3}$, eosinophils $\left.=100 / \mathrm{mm}^{3}\right)$, serum iron $=59 \mathrm{mg} / \mathrm{dL}$, and serum ferritin $=326 \mathrm{mg} / \mathrm{dL}$.

No intramural thrombi or myocardial dysfunction was detected by Doppler echocardiogram. The electrocardiogram was normal, and a chest X-ray confirmed left pleural effusion.

Diuretics and simvastatin (40 mg/day) were administered.

Nephrotic syndrome or liver failure were ruled out as causes of hypoalbuminemia after measurement of 24-h proteinuria $(0.61 \mathrm{~g}$ and $0.78 \mathrm{~g}$ ) and liver function (prothrombin time $=85 \%$ and factor V activity $=100 \%$ ). Hepatitis B and C serological tests were negative. Fecal chromium-51labeled albumin revealed a relevant loss of $600 \mathrm{~mL}$ in 24 $\mathrm{h}$ (normal values $<14 \mathrm{~mL}$ in $24 \mathrm{~h}$ ), confirming proteinlosing enteropathy as the diagnosis.

Once we had determined a protein-losing enteropathy, its etiology was investigated. There was no evidence of blood, fat, white blood cells, or parasites in the patient's feces. Serology for HIV, antinuclear antibodies, and antiendomysial antibodies was negative. Serum immunoglobulins were within normal ranges. Abdominal computed tomography revealed moderate ascites and mild hepatosplenomegaly. The ascitic and pleural liquids did not exhibit the features of a transudate. A small benign gastric ulcer with presence of Helicobacter pylori was revealed by endoscopy with gastric biopsy. Colonoscopy was performed, and 4 small tubulovillous polyps with high-grade dysplasia were completely excised.

Contrast radiography revealed increased transit time of the small intestine and nonspecific alterations in the mucosa. Enteroscopy showed no alterations. Multiple duodenal and jejunal biopsies revealed a chronic nonspecific enteritis. Intestinal biopsies (small bowel and colon) were negative by red-Congo stain.

Persistent thrombocytosis after improvement of the lesions in the patient's toes and inflammatory activity markers led to cytogenetic analysis of the bone marrow, which revealed large megakaryocytes and platelet debris. These findings suggested essential thrombocythemia. Hydroxyurea $1 \mathrm{~g} /$ day was administered to the patient.
The patient's general condition worsened, with intense asthenia, edema unresponsive to diuretics; the patient developed dyspnea and suddenly died. Necropsy did not reveal the cause of death, but histological study of the gastrointestinal tract showed findings consistent with eosinophilic gastroenteritis (Figure 2).

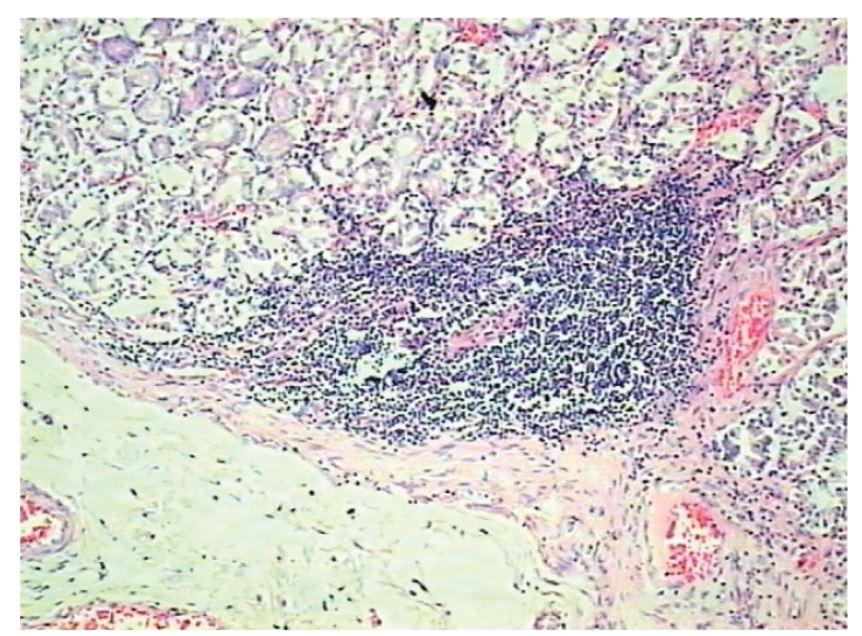

Figure 2 - Histological study of gastrointestinal tract. Note an abundant eosinophilic infiltration of the mucosa layer and the presence of lymphocytes in the corion. These findings are consistent with eosinophilic gastroenteritis (HE - 200x)

Protein-losing enteropathy is an unusual manifestation of some diseases, particularly gastrointestinal disorders, which may be associated with severe hypoalbuminemia. A gastrointestinal disorder should be considered when the regular causes of hypoalbuminemia such as malnutrition, nephrotic syndrome, and chronic liver disease are ruled out. Protein-losing enteropathy diagnostic studies consist of measuring fecal \pm 1 -antitrypsin or fecal chromium-51labeled albumin, and its main causes are ulcerative lesions of the gastrointestinal tract, nonulcerative lesions in the mucosa, and lymphatic diseases. In this case, diagnosis was provided by scintigraphy, but the etiology was only revealed at autopsy: findings of the detailed histological study of the gastrointestinal tract were consistent with eosinophilic gastroenteritis, a rare cause of enteric loss of protein. ${ }^{6}$

Eosinophilic gastroenteritis is a rare disease that typically manifests itself between the third and fifth decades of life. It is slightly more prevalent in men. Its etiology is unknown, and its pathogenesis poorly understood. Recent data suggest that in this disorder, eosinophils may directly damage the gastrointestinal tract wall by releasing granules containing cationic proteins and leukotrienes. ${ }^{7}$

Final diagnosis is provided by the following criteria: presence of gastrointestinal symptoms, eosinophilic infil- 
tration in 1 or more biopsied areas, absence of eosinophilic involvement in other organs, and absence of a parasite infection.

Approximately 50\% of patients have a past or family history of allergy (including atopy, asthma, or nasal polyps) or history of food intolerance or food allergy. The stomach and the small bowel are the most commonly involved organs.

Pathophysiological and clinical classification of eosinophilic gastroenteritis has been proposed according to the layer of the gut wall mostly affected: mucosa, muscle layer, or subserosal tissues. ${ }^{4}$ The most prevalent form affects mostly the mucosal (and submucosal) layers, and its symptoms typically include colicky pain, nausea, vomiting, diarrhea, and weight loss. Iron-deficiency anemia is common, and malabsorption syndrome may be present; protein-losing enteropathy may occur in $20 \%$ to $30 \%$ of cases. ${ }^{4,8}$ The musclelayer disease typically presents with pyloric or upper intestinal obstruction, such as colicky abdominal pain, nausea, and vomiting. It is associated with a thickened and rigid bowel. The rarest form of eosinophilic gastroenteritis is the serosal disease. It usually affects all layers of the bowel, and patients may present eosinophilic ascites with serosal and visceral peritoneal inflammation. The ascitic fluid is often a sterile exudate with a high eosinophil count.

In this case, the ascitic fluid was a transudate, suggesting that it was associated with severe hypoalbuminemia and not with the disease itself. Although the association of pleural effusion with the disease is only reported in very few cases, ${ }^{2}$ the pleural effusion of this patient should be associated with his hypoalbuminemia.

The radiographic changes found in eosinophilic gastroenteritis vary and are not specific. ${ }^{9}$ The gastric and small intestine folds can be thickened, with or without nodular filling defects, and the small intestine may be also dilated. Occasionally, the ileum resembles the jejunum because of increased thickness of the ileal folds. These changes may be absent in at least $40 \%$ of patients. Computed tomography may reveal a thickened intestinal wall and localized mesenteric lymphadenopathy, with serosal involvement and with the presence of ascitic fluid.

Endoscopy examination may show a normal mucosa or prominent mucosal folds, hyperemia, ulceration, and presence of nodules. Findings at laparoscopy vary from hyperemia of the intestinal loops to a picture resembling peritoneal carcinomatosis.

Upper GI endoscopy can show a normal mucosa or prominent folds, hyperemia, ulceration, and nodules of the gastrointestinal mucosa. Laparoscopy may show hyperemia of bowel folds and a pattern similar to that of peritoneal carcinomatosis.
Sometimes gastrointestinal mucosa biopsies reveal no abnormality. In certain situations, laparoscopic-guided biopsies are indicated to fully evaluate the thickness of the bowel wall. ${ }^{10}$ Histological analyses reveal edema and an inflammatory infiltrate consisting mainly of eosinophils arranged in chains and fully infiltrated into the submucosal layer. In this report, the gastric and intestinal biopsies did not show any specific abnormality. Diagnosis of eosinophilic gastroenteritis with essential thrombocytosis was made only at necropsy when a large portion of the gastrointestinal tract was carefully studied. There is no curative strategy to treat eosinophilic gastroenteritis; however, prednisone can be used to control the inflammation and suppress the episodes of disease attacks. The maintenance dose of prednisone can prevent symptoms from appearing. Other corticosteroids are being investigated. Azathioprine and other immune suppressors have been successfully used in some cases, but the side effects of these drugs are frequently responsible for discontinuation of therapy.

As for the hematological findings of this disease, iron deficiency anemia is frequent, and peripheral eosinophilia is present in up to $80 \%$ of the cases. Nevertheless, platelet abnormalities have not been described. In this case, eosinophilia and iron deficiency anemia were not observed, in contrast to the presence of severe thrombocytosis.

The elevated platelet count could be a reactive thrombocytosis to the inflammatory process in the patient's foot fingers, but the persistence of an elevated platelet count (above 1 million per $\mathrm{mm}^{3}$ ) after improvement of the clinical picture and a reduction of serum inflammatory marker levels suggested essential thrombocythemia, a myeloproliferative disease. The presence of digital ischemia, a common manifestation of this disease, and the good response to the use of an antiplatelet agent reinforced the hypothetical diagnosis. The presence of giant megakaryocytes and platelet debris in the bone marrow are compatible with the diagnosis of essential thrombocytosis. ${ }^{11}$ The patient's age was within the age range in which most cases are diagnosed (50-60 years). The development of digital ischemia was probably facilitated by risk factors related to atherosclerosis, such as dyslipidemia, hypertension, and smoking history. High-risk patients or those with a previous history of vascular events such as cerebrovascular or digital ischemia should receive aggressive platelet depletion therapy in order to maintain platelet count below 500,000 per $\mathrm{mm}^{3}$. Another possible therapy is plateletpheresis, which can be used to quickly reduce the platelet count and control the symptoms. A low dose of an antiplatelet agents can prevent thrombotic events in the acute and subacute phases, but its chronic use in asymptomatic patients has not yet been evaluated. ${ }^{5}$ In the present case, the patient was initially 
treated with aspirin due to the acute thrombotic event. Later, when the primary nature of the thrombocytosis had been defined, hydroxyurea was administered.

Essential thrombocythemia is not the cause of protein- losing enteropathy. The relationship between this disease and eosinophilic gastroenteritis is not well understood. We present a unique case of association between these two diseases.

\section{REFERENCES}

1. Binder HJ. Distúrbios da Absorção. In: Braunwald E, Fauci AS, Kasper DL, Hauser SL, Longo DL, Jameson JL, editors. Medicina Interna. $15^{\text {a }}$ ed. McGraw-Hill; 2001. p. 1777.

2. Talley NJ. Eosinophilic Gastroenteritis. In: Feldman M, Friedman LS, Sleisenger MH. Sleisenger \& Fordtran's Gastrointestinal and Liver Disease. $7^{\text {a }}$ ed. Saunders; 2002. p. 1972.

3. Cello JP. Eosinophilic gastroenteritis—a complex disease entity. Am J Med. 1979;67:1097-104.

4. Talley NJ, Shorter RG, Phillips SF, Zinsmeister AR. Eosinophilic gastroenteritis: a clinicopathological study of patients with disease of the mucosae, muscle layer, and submucosal tissues. Gut. 1990;31:548.

5. Schafer AI. Thrombocytosis. N Engl J Med. 2004;350:1211-19.

6. Greenberger NJ, Tennebaum JI, Ruppert RD. Protein-losing enteropathy associated with gastrointestinal allergy. Am J Med. 1967;43:777-84.
7. Rothenberg ME. Eosinophilic gastrointestinal disorders. J Allergy Clin Immunol. 2004;113:11-28.

8. Kalantar SJ, Marks R, Lambert JR, Badov D, Talley NJ. Dyspepsia due to eosinophilic gastroenteritis. Dig Dis Sci. 1997;42:2327-32.

9. 10. MacCarty RL,Talley NJ. Barium studies in diffuse eosinophilic stroenteritis. Gastrointest Radiol 1990;15(3):183-7.

11. Clegg-Lamptey JN, Tettey Y, Wiredu EK, Kwawukume EY. Eosinophilic enteritis-a diagnostic dilemma. West Afr J Med. 2002;21:258-9.

12. Barbui T, Barosi G, Grossi A, Gugliotta L, Liberato LN, Marchetti M, et al. Practice guidelines for the therapy of essential thrombocythemia. A statement from the Italian Society of Hematology, the Italian Society of Experimental Hematology and the Italian Group for Bone Marrow Transplantation. Haematologica. 2004;89:215-32. 